\title{
Allee dynamics generated by protection mutualisms can drive oscillations in trophic cascades
}

\author{
Manuel A. Morales • William F. Morris • \\ William G. Wilson
}

Received: 23 May 2007 / Accepted: 31 August 2007 /Published online: 2 October 2007

(C) Springer Science + Business Media B.V. 2007

\begin{abstract}
Understanding the relative effect of top predators and primary producers on intermediate trophic levels is a key question in ecology. Most previous work, however, has not considered either realistic nonlinearities in feedback between trophic levels or the effect of mutualists on trophic cascades. Here, we develop a realistic model for a protection mutualism that explicitly includes interactions between a protected herbivore and both its food plant and generalist predators. In the absence of protection, herbivores and plant resources approach a stable equilibrium, provided that predation is not so high as to cause herbivore extinction. In contrast, adding protection by mutualists increases the range of dynamical outcomes to include unstable equilibria, stable and unstable limit cycles, and heteroclinic orbits. By reducing the impact of predators, protection by mutualists can allow herbivores to exert strong negative effects on their host plants, which in turn can lead to repeated cycles of overexploitation and recovery. Our results indicate that it may be essential to consider protection mutualisms to understand the dynamics of trophic cascades. Conversely, it may be essential to explicitly include dynamical feedback between plants and herbivores to fully understand the population and community dynamical consequences of protection mutualism.
\end{abstract}

Keywords Hopf bifurcation · Host-plant quality ·

Limit cycle · Protection mutualism · Trophic cascade

M. A. Morales ( $\varangle)$

Department of Biology, Williams College,

Williamstown, MA 01267, USA

e-mail:mmorales@williams.edu

W. F. Morris • W. G. Wilson

Department of Biology, Duke University,

Durham, NC 27708, USA

\section{Introduction}

Since its introduction, Hairston, Smith and Slobodkin's (Hairston et al. 1960) hypothesis of top-down trophic regulation (HSS) has played a key role in extending models of pairwise species interactions to a community context. HSS is based on the assumption of alternating predator and food limitation for sequentially lower trophic levels. From this assumption, HSS predicts a positive effect of top predators on primary producers in communities with three trophic levels but a negative effect of top predators on primary producers for communities with four trophic levels (Fretwell 1977).

More recently, theoretical models and empirical studies have refined HSS to include greater realism by evaluating the effect of predator diversity on the magnitude of topdown control (Finke and Denno 2004; McCann et al. 1998) and by considering the effect of omnivory (feeding between nonadjacent trophic levels) on community dynamics (Diehl and Feißel 2000). Overall, there has been a shift away from categorizing communities as controlled by either bottomup or top-down forces and toward evaluating variability in the relative effect of predator or resource limitation on community dynamics (Hunter and Price 1992).

Despite these advances, significant gaps remain in our understanding of trophic cascades. For example, although there has been a call to develop a community perspective on mutualism (Stanton 2003), few studies have addressed the effect of mutualism on the dynamics of trophic cascades. Empirical studies that have considered the effect of plant and herbivore mutualists on trophic cascades (Knight et al. 2006; Styrsky and Eubanks 2007) suggest that mutualism can be an important, albeit understudied, component of trophic dynamics. We are unaware, however, of any theoretical studies that have considered the effect of mutualism on the interactions between higher- and lower-level consumers- 
e.g., the inhibition of top predators caused by protection mutualists.

In protection mutualisms, a protector species benefits a host species by removal of the host's natural enemies in return for a resource reward (Bronstein and Barbosa 2002). Protection mutualisms are geographically and taxonomically diverse, and include interactions between cleaner species (fish or arthropods) and their hosts, between ants and plants that produce extrafloral nectar or other food rewards for the ants, and between ants and herbivores. Protection mutualisms are common adjuncts of trophic cascades (Halaj and Wise 2001; Schmitz et al. 2004), perhaps because protection mutualists can generate simplified local communities (Risch and Carroll 1982; Wimp and Whitham 2001) in which trophic cascades are predicted to be more likely (Hunter and Price 1992).

According to HSS, predators may prevent consumers from greatly depressing their resources. By reducing predation on bottom consumers, protection may facilitate resource overexploitation, raising the possibility that protection mutualism may lead to cyclic dynamics in which a period of consumer population growth and resource overexploitation is followed by a period of consumer decline and resource recovery, which can then initiate a new cycle, and so on. Although most previous studies have focused on the effect of the number of trophic levels on equilibrium densities (e.g., Power 1992), feedback between trophic levels can also generate nonequilibrial dynamics (Hastings and Powell 1991).

Given the potential importance of mutualism in trophic dynamics and the intrinsically trophic nature of protection mutualism, we develop a model to evaluate how protection mutualism influences the dynamics of multi-trophic-level interactions. Our model incorporates generalist top predators and protectors, immigration of the top predator and protector to a bottom consumer (e.g., a herbivore) with emigration in proportion to its density, and feedback from the bottom consumer to the basal resource (e.g., a plant). We assume that protectors and predators respond to herbivores on a behavioral time scale and that their densities rapidly equilibrate to the current herbivore density. To assess the role of protection mutualism for dynamics of consumer populations, we compare the range of dynamical possibilities in a simple foodchain model with and without protectors. In the Discussion, we place our results in the context of past theoretical work on trophic cascades, on the impact of mutualism on community stability, and on the likelihood of herbivore fluctuations driven by feedback to host-plant quality.

\section{A trophic cascade model with protection mutualism}

A basic model of a three-level trophic cascade with a protection mutualism requires four components: a basal resource, a consumer of that resource, a predator of that consumer, and a protector that modulates predation on the consumer (Fig. 1). A number of interactions fit this basic template. For example, in ant-plant interactions, the four components, respectively, are abiotic nutrients, plants, herbivores, and ants, whereas in host-cleaner interactions, they are basal prey, host, parasite, and cleaner. Although relatively little is known about the benefits and dynamics of the participants in many of these interactions (Bronstein and Barbosa 2002), ant-herbivore protection mutualisms are a particularly well-studied category of protection mutualism, so we use a plant-herbivorepredator-ant system to motivate our model.

Ant-tended herbivores are insects (e.g., aphids and caterpillars) that excrete or secrete sugar rewards in return for protection from predators (Buckley 1987; Cushman and Whitham 1989; Morales 2000b; Pierce and Easteal 1986). Often the rewards are metabolic byproducts that involve little or no cost to produce. The ant species that tend herbivores and the predators of those herbivores are typically generalists, even in cases where the ant-tended herbivores are obligately dependent on ant protection (Buckley 1987; Devries 1991; Way 1963). Ant-protected herbivores are usually tended in aggregations and are characterized by limited mobility and ant-dependent host-plant selection (Axén and Pierce 1998; Dyck et al. 2000; Morales 2002). As a consequence, an ant-tended herbivore aggregation is largely restricted to a single plant chosen by a foundress. For a given plant or small patch of plants, the processes controlling local ant and predator densities are largely behavioral and thus operate on a faster time scale than the dynamics of herbivore density (Morales 2000a, b) or hostplant quantity or quality.

Given the typically limited mobility of ant-tended herbivores, we model the dynamics of birth and death of herbivores on a single plant, but we assume that generalist ant and predator populations on the plant are open, with immigration and emigration dependent on behavioral processes that are, in part, a response to the local herbivore density. We assume that regional ant and predator densities

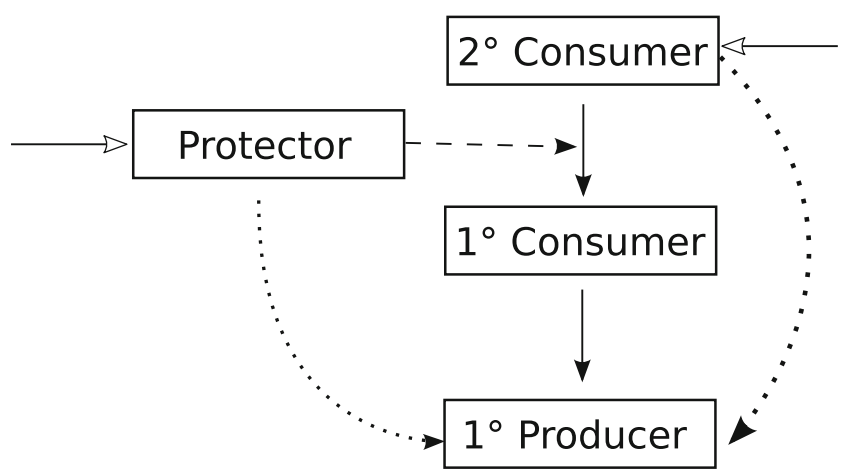

Fig. 1 Schematic diagram of protection mutualism illustrating direct and indirect effects in a trophic cascade. Solid lines are trophic interactions; dashed lines are behavioral interactions; dotted lines are indirect interactions; open arrows represent immigration 
are largely determined by resources other than those provided by the local herbivore population (as is reasonable for highly generalist species). We also assume that the protection mutualism is strictly indirect: mutualists benefit herbivores solely via their effect on local predator density and neither consume herbivores nor benefit them in any other way (e.g., by increasing feeding rates). Finally, feeding by the local herbivore population influences the size or the nutritional quality of the single plant on which it is located.

Defining $M, P$, and $H$ as the densities of protectors (synonymously, "mutualists"), predators, and herbivores on the plant and $Q$ as the quantity or quality of the plant from the perspective of herbivores, our full model of the food web is

$$
\begin{aligned}
& \frac{d M}{d t}=I_{M}+\frac{\alpha H}{1+\beta H}-\delta_{M} M \\
& \frac{d P}{d t}=I_{P}-\frac{1+\gamma M}{1+\varphi H} \delta_{P} P \\
& \frac{d H}{d t}=r_{H} Q H-\varepsilon P H \\
& \frac{d Q}{d t}=r_{Q} Q(1-Q)-\eta H Q
\end{aligned}
$$

In the absence of herbivores, $I_{M}$ mutualists immigrate to the plant per unit time, and the per-capita emigration rate is $\delta_{M}$. The equilibrium density of mutualists in the absence of herbivores is therefore $I_{M} / \delta_{M}$. The link to the other populations is provided by a saturating increase in mutualist recruitment in response to herbivores, with a maximum increase of $\alpha / \beta$ at high herbivore density and half saturation when $H=1 / \beta$. Saturating mutualist recruitment assumes that the availability of mutualists is limited (Breton and Addicott 1992; Cushman and Addicott 1989; Cushman and Whitham 1991; Morales 2000a,b) and produces the realistic result that run-away population growth driven by the mutualism cannot occur (cf. May 1978; Vandermeer and Boucher 1978; Wolin and Lawlor 1984). In the absence of herbivores and their mutualists, predators arrive at and leave host plants in a manner similar to mutualists but at rates controlled by $I_{P}$ and $\delta_{P}$, respectively. The predator population is coupled to the food web in two different ways. First, the predator's emigration rate declines to zero (at a rate scaled by $\phi$ ) as the density of its prey, the herbivores, increases. Second, mutualists protecting herbivores increase the predator's rate of emigration from the plant, at a rate scaled by $\gamma$. Therefore, the magnitude of benefit to herbivores is proportional to $\gamma$. Herbivores suffer mortality in proportion to predators at a per-capita rate $\varepsilon$.
Equation 1d could represent logistic growth of plant biomass with type I consumption by herbivores, or herbivore-induced changes in plant quality (Morris and Dwyer 1997). In either case, $Q$ is scaled so that its maximum is 1 . In the absence of herbivores, $Q$ increases at a rate determined by $r_{Q}$. $Q$ declines with increasing herbivore density at a rate governed by $\eta$. If $Q=1$, herbivores are born at a per-capita rate $r_{H}$, but herbivore birth declines linearly as $Q$ declines.

To reduce the number of parameters, we nondimensionalize Eqs. $1 \mathrm{a}-1 \mathrm{~d}$ by applying the definitions $\tau \equiv t \delta_{P}$, $M^{\prime} \equiv \delta_{P} M / I_{M}, \quad P^{\prime} \equiv \varepsilon P / \delta_{P}, \quad H^{\prime} \equiv H \beta, \quad m_{P} \equiv I_{P} \varepsilon / \delta_{P}^{2}$, $g \equiv \gamma I_{M} / \delta_{P}, \quad k \equiv \varphi / \beta, \quad a \equiv \alpha / \beta I_{M}, \quad d_{M} \equiv \delta_{M} / \delta_{P}$, $R_{H} \equiv r_{H} / \delta_{P}, R_{Q} \equiv r_{Q} / \delta_{P}$, and $h \equiv \eta / \beta \delta_{P}$, yielding the simplified set of equations

$\frac{d M}{d \tau}=1+\frac{\alpha H}{1+H}-d_{m} M$

$\frac{d P}{d \tau}=m_{P}-\frac{1+g M}{1+k H} P$

$\frac{d H}{d \tau}=R_{H} Q H-P H$

$\frac{d Q}{d \tau}=R_{Q} Q(1-Q)-h H Q$

(where we have dropped primes for clarity).

\section{Community dynamics in the absence of mutualism and with rapidly equilibrating predators}

We first explore the dynamics of the community in the absence of protection mutualism. Specifically, we eliminate Eq. 2a and set $M=0$ in Eq. 2b. Furthermore, because the behavioral timescale at which predators arrive at and leave plants is likely to be much faster than the time scale at which local herbivore density and plant quantity or quality change, we assume that the predator density on the plant rapidly equilibrates to local herbivore density. Mathematically, we assume that the predator continuously attains its "quasiequilibrium" value, $P^{*}$, obtained by setting Eq. $2 \mathrm{~b}$ to zero (with $M=0$ ). Replacing $P$ with $P^{*}$ in Eq. 2 c yields a herbivore-plant model with implicit predation effects:

$$
\begin{aligned}
& P^{*}=m_{P}(1+k H) \\
& \frac{d H}{d \tau}=R_{H} Q H-P^{*} H \\
& \frac{d Q}{d \tau}=R_{Q} Q(1-Q)-h H Q
\end{aligned}
$$

In the Appendix, we show that any biologically realistic internal equilibrium of Eq. 3 will be strictly stable. Therefore, we conclude that if local predator density closely tracks local herbivore density, then the negative feedback of 
herbivores on plants embodied in Eqs, $2 \mathrm{c}$ and $2 \mathrm{~d}$ will not cause long-term fluctuations in plants, herbivores, and predators in the absence of protectors (Fig. 2).

\section{The effect of protection mutualists on community dynamics}

Retaining the assumption of rapidly equilibrating predators, we next add protectors. As with predators, we assume that local protector density closely tracks local herbivore density (justified by the assumption that the dynamics of protectors, like that of predators, operates on a fast behavioral time scale). Specifically, assuming $d M / d \tau=0$, we solve for the quasiequilibrium mutualist density $M^{*}$ and substitute it into the right-hand side of Eq. $2 \mathrm{~b}$. Then, assuming quasiequilibrium for the predators, we obtain $P^{*}$ and substitute the result for $P$ in Eq. 2c. This series of assumptions yields a model of herbivore/plant dynamics with implicit predators and mutualist protectors:

$$
\begin{aligned}
M^{*} & =\left(1+\frac{a H}{1+H}\right) \frac{1}{d_{M}} \\
P^{*} & =m_{P} \frac{1+k H}{1+g M^{*}} \\
\frac{d H}{d \tau} & =R_{H} Q H-P^{*} H \\
\frac{d Q}{d \tau} & =R_{Q} Q(1-Q)-h H Q
\end{aligned}
$$

The resulting model is highly nonlinear, reducing the utility of standard linear stability analysis, so instead, we used bifurcation diagrams and numerical solutions to assess the existence and stability properties of the equilibria, as well as the dynamics away from equilibria (Ermentrout 2002).

Protection mutualists increase the range of dynamical possibilities beyond those predicted by the mutualist-free model. For some parameter combinations, we see an unstable, low-density herbivore equilibrium, as is often seen in models of obligate mutualism (e.g., May 1978; Vandermeer and Boucher 1978; Wolin 1985; Morris et al. 2003). We also observe equilibria undergoing subcritical and supercritical Hopf bifurcations (Kuznetsov 1995) and the existence of unstable and stable limit cycles. That is, unlike the model without protection mutualism (Eq. 3), the model with implicit mutualists (Eq. 4) can produce sustained oscillations in herbivores and plants (Fig. 2).

The destabilizing effect of protectors is caused by an Allee effect in the herbivores - specifically, an initial decrease in the herbivore's per-capita death rate with increasing herbivore density. This Allee effect means that herbivores will recover relatively slowly when depressed to low density, thus introducing a lag that allows $Q$ to increase before herbivores recover, which then allows herbivores to overexploit $Q$ and then decline to low levels, reinitiating the cycle.
Fig. 2 Population trajectories (solid lines) of herbivores $(H)$ and plant quantity/quality $(Q)$ for the model with implicit predators and without protectors (Eq. 3) (top) compared to the model with protectors (Eq. 4) (bottom). Note similar results (dashed lines - not visible in the top panels because lines overlap) obtained using the full model (Eqs. 2a-2d). Parameter values are set to the baseline values but with $R_{\mathrm{H}}=2$
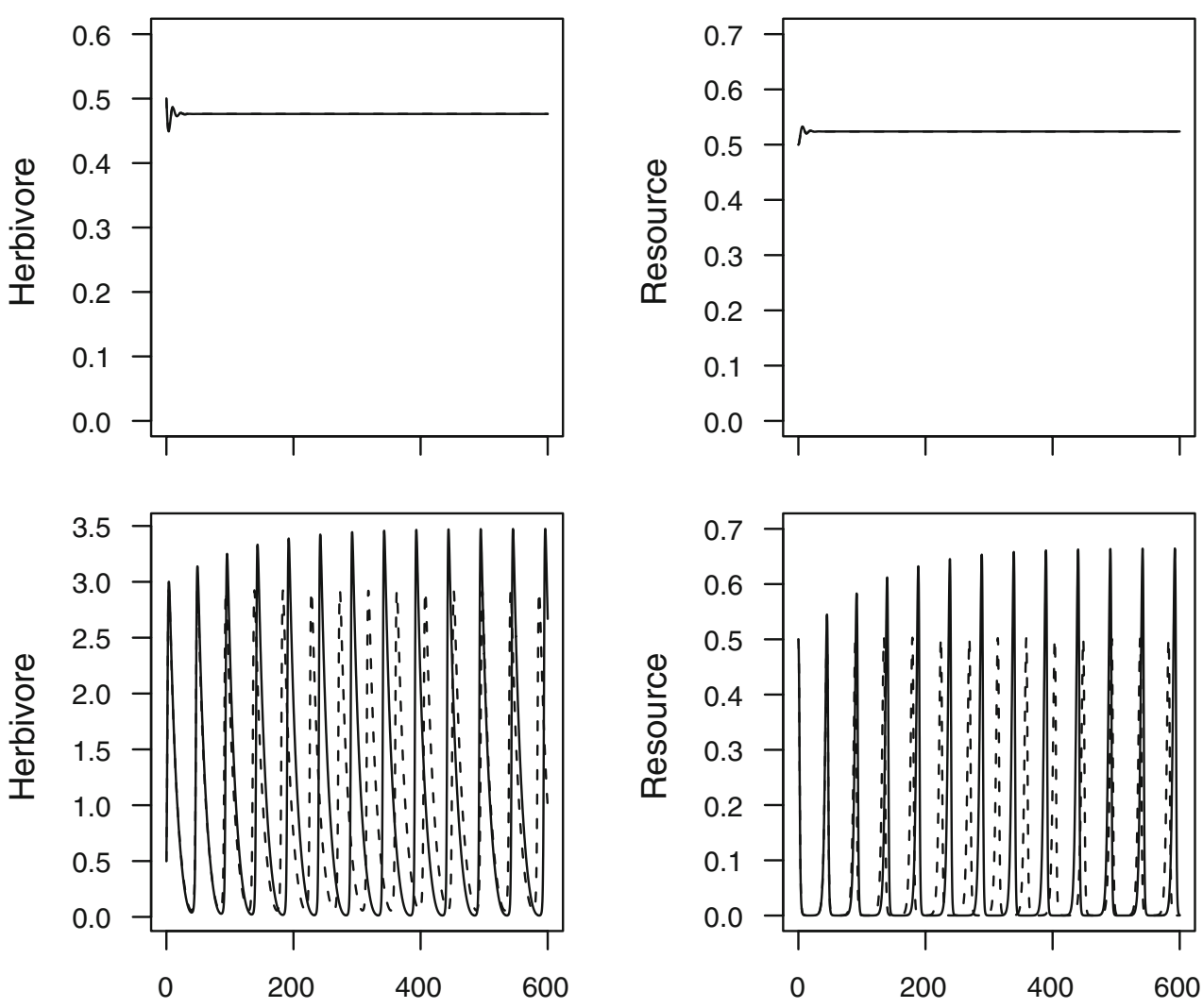

Time

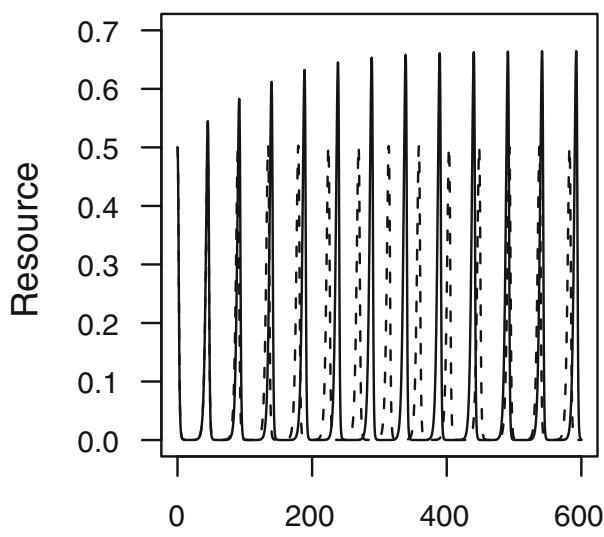

Time 
Comparing the model with predators at quasiequilibrium but without protectors (Eq. 3) vs that with protectors (Eq. 4), the herbivore's per-capita death rate is lower in the presence of protectors (Fig. 3). More importantly, the per-capita death rate increases linearly with herbivore density in the absence of protectors but shows an initial decrease at low herbivore density in the presence of protectors. The herbivore's percapita death rate initially declines because increasing protector immigration (Eq. 2a) causes predator emigration to increase (Eq. 2b), thus decreasing the quasiequilibrium predator density (Fig. 4). As herbivore density continues to increase, saturation of protector immigration allows quasiequilibrium predator density to track herbivore density so that the per-capita herbivore death rate again increases (see Appendix for the stability conditions for a simple analog of Eq. 4 with an Allee effect).

To further explore the conditions for cyclic dynamics to occur, we varied the parameters in Eq. 4 one at a time while fixing all other parameters at default values (Table 1) that we chose on the basis of our knowledge of protection mutualisms and of a preliminary exploration of parameter space to locate regions where dynamics change. Increasing the maximum herbivore birth rate $\left(R_{H}\right)$ shifts the number of positive equilibria from 0 to 2 (near $R_{H}=0.2$ in Fig. $5 \mathrm{a}$ ) to 1 . In the low $R_{H}$ region, where two positive equilibria exist, the mutualism is strongly obligate - the herbivore's intrinsic growth rate is too low for it to be able to persist at the equilibrium density of mutualists that would be present on an herbivore-free plant, and the lower equilibrium is thus unstable (see Appendix). Initial densities above the lower equilibrium will be attracted to the upper equilibrium, and initial densities below the lower equilibrium will decline to

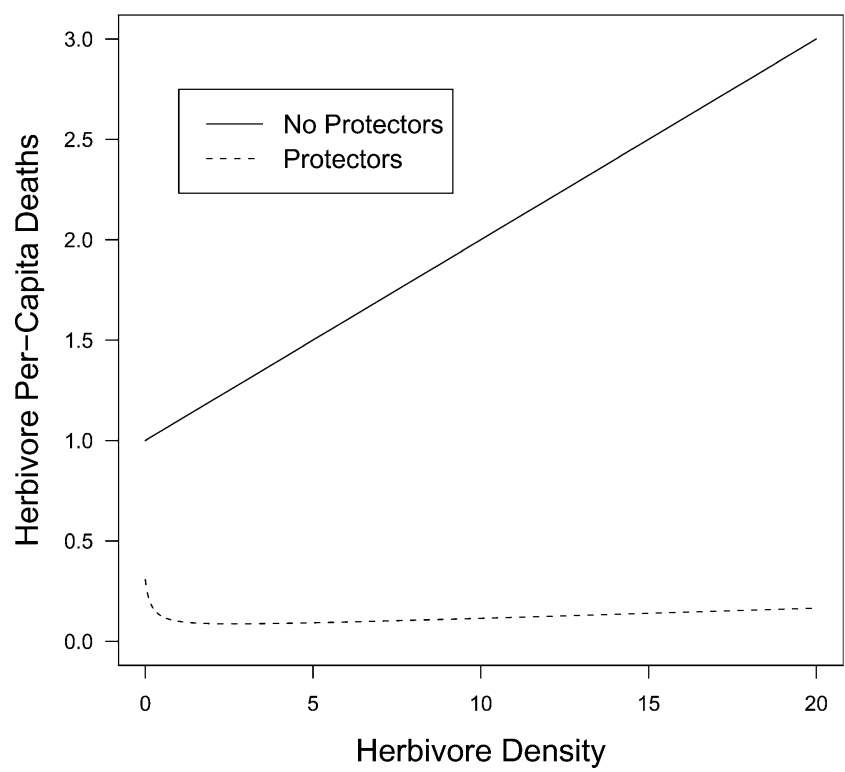

Fig. 3 The herbivore per-capita death rate at the baseline parameter values

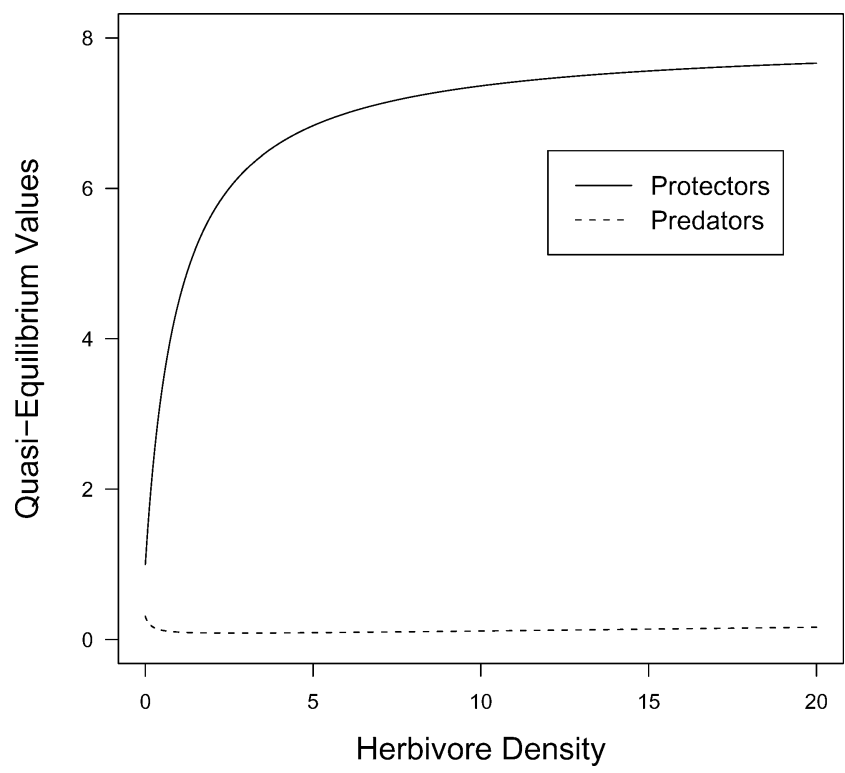

Fig. 4 Quasiequilibrium densities of protectors and predators as a function of herbivore density in the model of Eq. 4, using baseline parameter values

zero (Fig. 6a). As $R_{H}$ increases further, it passes from a zone with a single stable equilibrium to a zone near $R_{H}=1.7$ in which a stable equilibrium and a stable limit cycle coexist, with an unstable limit cycle separating the two basins of attraction. These situations are depicted by phase plane plots in Fig. 6b,c. As $R_{H}$ increases even further, the unstable limit cycle collides with the stable equilibrium in a subcritical Hopf bifurcation (i.e., the stable equilibrium and the unstable limit cycle with which it ultimately collides coexist on one side of the bifurcation) near $R_{H}=1.8$, beyond which the equilibrium is unstable and the stable limit cycle is the only attractor (see phase plane plot in Fig. 6d).

In the zone with a single, unstable equilibrium, changing the negative effect of herbivores on host plants $(h)$ so that it is sufficiently lower or higher than its default value stabilizes the system [Fig. 5b, which shows a subcritical Hopf bifurcation at $h \approx 0.6$ and a supercritical Hopf bifurcation (i.e., the stable equilibrium and the stable limit cycle emanating from it exist on opposite sides of the bifurcation point) at $h \approx 4$ ]. Low values of $h$ are stabilizing because feedback from herbivores to plants is weak, and hence, herbivore density is controlled by predation, whereas high values of $h$ are stabilizing because $Q$ can never attain sufficiently high levels to allow herbivores to experience high population growth rates. In contrast, decreasing $R_{Q}$ (the recovery rate of $Q$ ) destabilizes the system by increasing the lag period before herbivores can again increase (Fig. 5c). Interestingly, increasing $g$ (the positive effect of mutualists on herbivores mediated through predator removal) is destabilizing (Fig. 5d). Cyclical dynamics are also associated with high values of the maximum mutualist recruitment rate 
Table 1 Default parameter values in the original model (Eqs. 1a-1d), which yield $m_{\tilde{\mathrm{N}}}=$ $1, k=0.1, g=2.25, a=7, d_{M}=1$, $R_{H}=1.75, R_{Q}=0.5$, and $h=0.5$ for the composite parameters in models $2 \mathrm{a}-4$

\begin{tabular}{|c|c|c|}
\hline Parameter(s) & Value & Comments \\
\hline$\delta_{P}, \delta_{M}, I_{P}, I_{M}$ & 1 & $\begin{array}{l}\text { Set immigration and emigration rates of mutualists and predators equal. Set } \\
\text { parameter value at } 1 \text { to facilitate the interpretation of nondimensionalized } \\
\text { parameter groupings }\end{array}$ \\
\hline$r_{H}$ & 1.75 & $\begin{array}{l}\text { Herbivore growth rates are large relative to the arrival and leaving rates of } \\
\text { mutualists and predators in the absence of herbivores }\end{array}$ \\
\hline$\varepsilon$ & 1 & $\begin{array}{l}\text { Set predation rate at } 1 \text { to facilitate the interpretation of nondimensionalized } \\
\text { parameter groupings }\end{array}$ \\
\hline$r_{Q}$ & 0.5 & Plant-quality recovers relatively slowly \\
\hline$\eta$ & 0.5 & $\begin{array}{l}\text { Set the carrying capacity }\left(r_{Q} / \eta\right) \text { of herbivores in the absence of either predators } \\
\text { or mutualists to } 1\end{array}$ \\
\hline$\alpha, \beta$ & 7,1 & $\begin{array}{l}\text { Recruitment increases strongly with herbivore density, and the half-saturation } \\
\text { point }(1 / \beta=1) \text { is high (Morales } 2000 \text { a) relative to the carrying capacity of } \\
\text { herbivores in the absence of predators or mutualists }\end{array}$ \\
\hline$\gamma, \phi$ & $2.25,0.1$ & $\begin{array}{l}\text { Predator density responds much more strongly to mutualist density than to } \\
\text { herbivore density (Morales, unpublished data) }\end{array}$ \\
\hline
\end{tabular}

( $\alpha$, Fig. 5e) and low values of the mutualist departure rate $\left(d_{M}\right.$, Fig. 5f), which indicates that increasing the potential range of the protection benefit is destabilizing. Finally, cycles are observed for high values of $m_{P}$ (Fig. $5 \mathrm{~g}$ ) and low values of $k$ (Fig. 5h), two parameters that control predation intensity. Because $m_{P}$ controls herbivore-independent predator arrival, predator density declines at low $m_{P}$ such that mutualists provide little benefit and the system resembles the stable, mutualist-free model (Eq. 3). In contrast, $k$ controls the herbivore-dependent recruitment of predators. If $k$ is large, predators increase quickly as herbivore density increases so that herbivore density can never get large enough to depress $Q$ and drive cycles.

Because strong obligate mutualism is characterized by a threshold density below which herbivores go extinct, the risk of extinction is especially pronounced for systems in which the upper equilibrium is unstable. Ultimately, if the oscillations about the upper equilibrium are relatively small, the oscillations occur inside the lower equilibrium's stable manifold and the oscillations persist (Fig. 7a). However, if the oscillations are large, the unstable manifold of the upper equilibrium joins the stable manifold of the lower equilibrium to produce a heteroclinic orbit - all other starting points result in extinction (Fig. 7b).

Finally, we note that when we numerically explored the number and stability properties of equilibria for both the model with predators and mutualists at quasiequilibrium (Eq. 4) and the full model (Eqs. 2a-2d) over broader regions of parameter space, we obtained similar frequencies of all possible dynamical outcomes (Table 2; see also dashed lines in Fig. 2). These results indicate that explicitly tracking the dynamics of arrival and departure of predators and mutualists does not alter the predicted dynamics of the full community once the dynamics of herbivores and plants have been made explicit.

\section{Discussion}

Our results show that adding a protection mutualist to a trophic cascade can produce community oscillations when three-way equilibria in the absence of the mutualism would be strictly stable. Therefore, sustained fluctuations of a consumer population driven by a dynamical interaction with its resource may be facilitated when the consumer is engaged in a protection mutualism with generalist protectors and preyed upon by mobile, generalist predators. This scenario describes well the mutualism between insect herbivores and their defenders, but it may also capture some essential features of other protection mutualisms. In the following paragraphs, we discuss our results in the light of previous theoretical studies of trophic cascades, of mutualism, and of herbivore population dynamics, and we outline several caveats that apply to our analysis.

Although the focus of trophic cascade theory has been on the factors that determine population density at equilibrium, results presented here and previous studies suggest that the dynamical properties of trophic communities may be equally important. For example, strong feedback between trophic levels can generate chaotic dynamics in a simple food-chain model (Hastings and Powell 1991). Implicit spatial dynamics of resources and consumers has also been shown to destabilize trophic communities (Nisbet et al. 1997). Here we add another factor, protection mutualism, that may destabilize trophic dynamics by introducing an Allee effect into predator-driven herbivore death rates.

The question of whether mutualism stabilizes or destabilizes communities has a checkered past, with some theoretical studies favoring each answer. Several overlapping factors have contributed to the divergent results. One is the use by different researchers of different definitions of "stability," including the absence of explosive population growth (Gause 
Fig. 5 Bifurcation plots showing maximum, minimum, and equilibrium herbivore density $(H)$ as a function of each of the parameters for the herbivorequality model, with other parameters held at default values (indicated by arrows; also see Table 1). Open circles identify the maximum and minimum values of an unstable limit cycle, and closed circles represent the maximum and minimum values of a stable limit cycle. A solid line indicates a stable equilibrium and a dashed line indicates an unstable equilibrium. A $R_{H}$, $\mathbf{B} h, \mathbf{C} R_{Q}, \mathbf{D} g, \mathbf{E} a, \mathbf{F} d_{M}, \mathbf{G}$ $m_{P}, \mathbf{H} k$
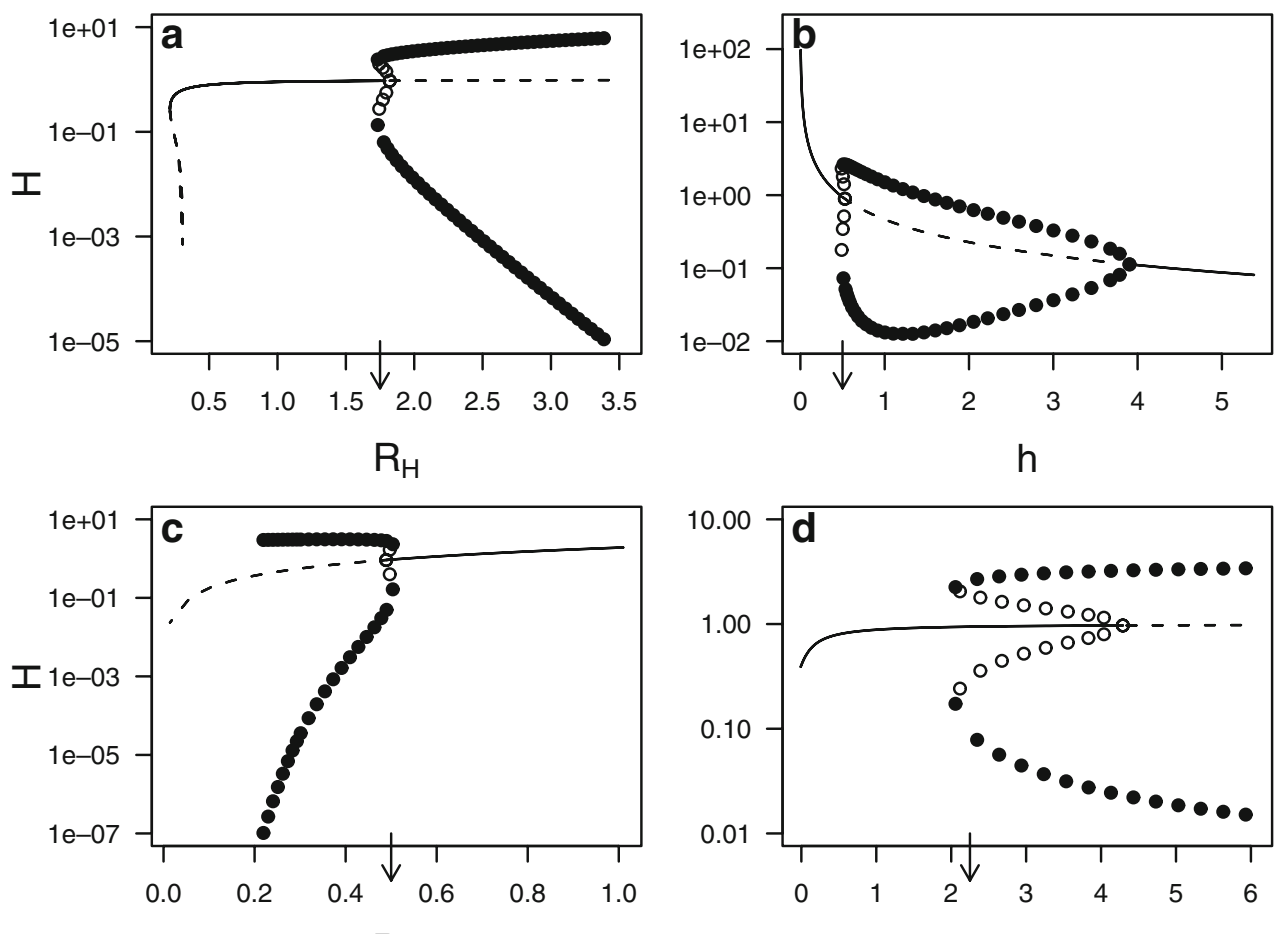

$\mathrm{R}_{\mathrm{Q}}$

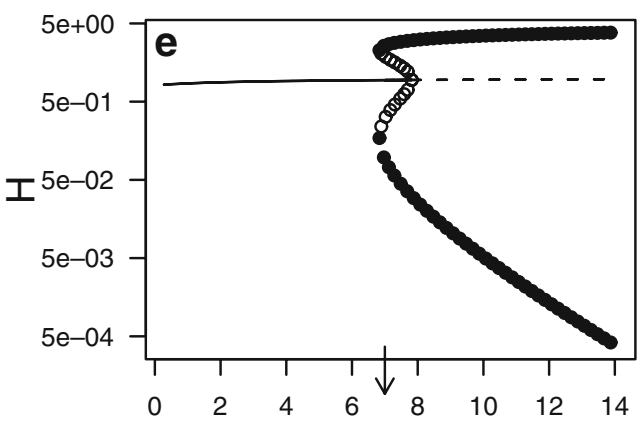

g

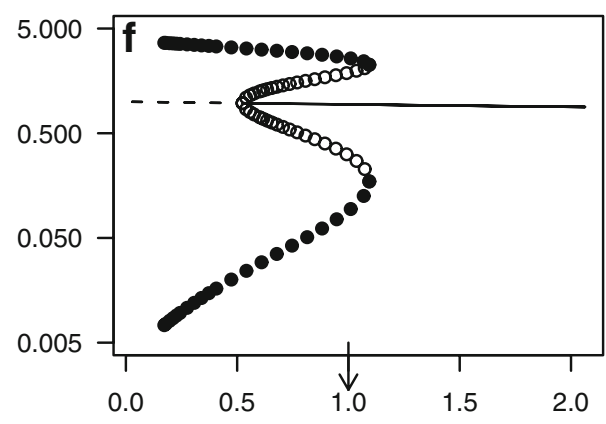

a

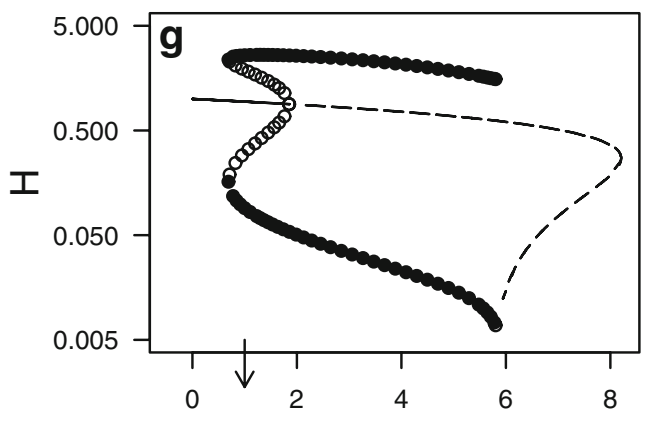

$m_{P}$

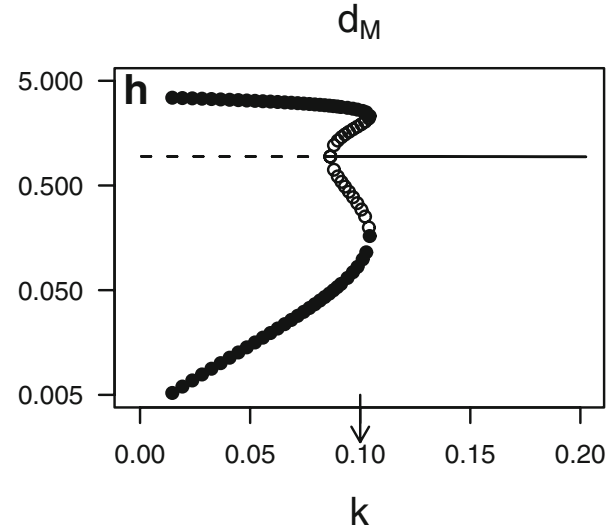

and Witt 1935; May 1976; Heithaus et al. 1980; Kooi et al. 2004), avoidance of extinction (Ringel et al. 1996), qualitative local stability of equilibria (May 1974), and a rapid rate of return to a stable equilibrium following a small perturbation (Addicott 1981; Wolin and Lawlor 1984; Ringel et al. 1996). A second factor is whether models assume (unrealistically) that benefits increase indefinitely as the density of a mutualistic partner increases (Gause and Witt 1935; May 1976; Heithaus et al. 1980; Addicott 1981; Ringel et al. 1996) or whether more realistic saturating benefits are assumed (May 1978; Vandermeer and Boucher 1978; Dean 1983; Wolin and Lawlor 1984; Armstrong 1987; Wright 
Fig. 6 Phase-plane diagrams illustrating the effect of increasing $R_{H}$ on herbivore $(H)$ and plant $(Q)$ dynamics. Unstable and stable equilibria are indicated by open vs closed circles, respectively. At low $R_{H}\left(\mathbf{A}, R_{H}=0.25\right)$, herbivores are obligately dependent on mutualists for protection from predators $(M)$. Densities outside or inside the stable manifold (thick gray line) of the lower equilibrium (a saddle point) will increase to the upper equilibrium or decrease to zero, respectively. The thick black line shows the unstable manifold of the lower equilibrium. As $R_{H}$ increases, the system switches to a single stable equilibrium (B, $R_{H}=1.0$ ), then to a coexisting stable equilibrium and a stable limit cycle separated by an unstable limit cycle (dashed gray line in $\mathbf{C}, R_{H}=1.78$ ), and finally to a stable limit cycle only for values of $R_{H}$ above the subcritical Hopf bifurcation $\left(\mathbf{D}, R_{H}=\right.$ 1.82). For $\mathbf{C}$, initial densities inside the unstable limit cycle will be attracted to the stable equilibrium, while initial densities outside it will be attracted to the stable limit cycle along the unstable manifold of the lower equilibrium (thick black line)
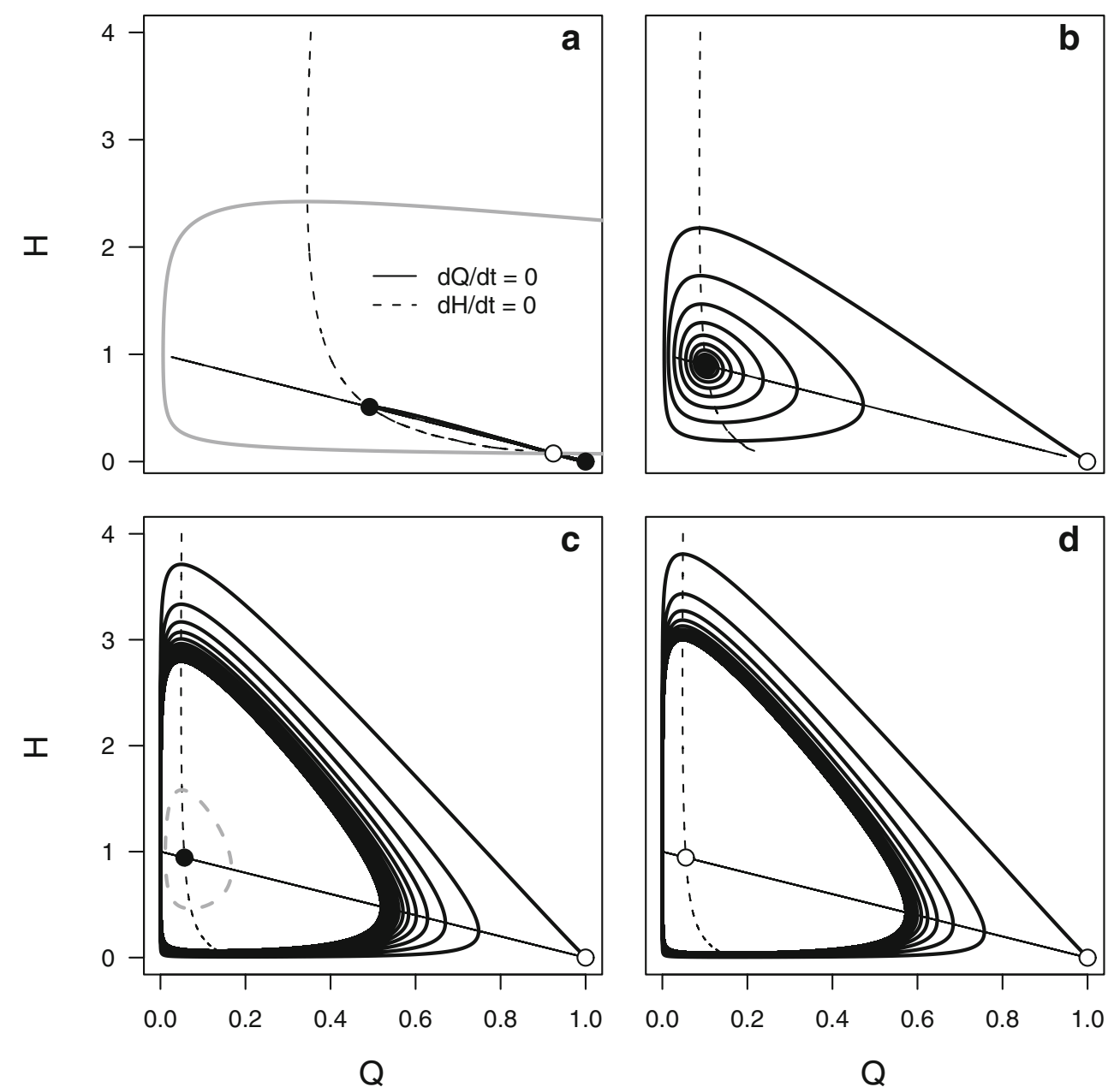

1989; Holland and DeAngelis 2001; Morris et al. 2003; Kooi et al. 2004). Yet a third factor is whether a model includes two or more species. Given this diversity of approaches, it is not surprising that no consensus has emerged. Indeed, rather than asking whether mutualism stabilizes or destabilizes communities in general, a far more relevant question is how specific types of mutualism may alter the dynamics of multispecies communities, as portrayed by tailored models that capture essential features of those mutualisms (cf. Kooi et al. 2004). In attempting to model the community centered on herbivores with protective mutualists, we have used realistic saturating benefits, have incorporated the interactive effects of three other species (including the fact that mutualists benefit herbivores indirectly through their effects on local predator density), and have allowed for differences in the time scales at which different processes in this community operate. Using this tailored model, we have shown that elevating the protection benefit that herbivores receive from mutualists can cause the community to undergo a transition from stability to sustained oscillations. We have also partially explored how other biological factors, such as the herbivores' birth rates, rates of decline and host-plant recovery, and mutualist and predator immigration and emigration rates, influence whether the systems with protection mutualism will be stable or oscillatory (Fig. 5). Estimating these parameters for real protection mutualisms and censusing the herbivore population over time would allow the predictive power of the model to be assessed.

In building a trophic model for protection mutualisms, we have also linked two areas of theoretical investigation that have largely developed in isolation from one another: mutualism models and models of herbivore population dynamics driven by induced changes in host-plant quality. The simplest hostplant quality models do not produce persistent herbivore fluctuations. For example, Edelstein-Keshet and Rausher (1989) found that continuous time models similar to Eqs. 2c and $2 \mathrm{~d}$ are stable. Indeed, it is easy to show that Eqs. $2 \mathrm{c}$ and $2 \mathrm{~d}$ with $P$ fixed, which is equivalent to a predator-prey model with logistically growing prey and a type I functional response, is strictly stable. More complex models that incorporate quality-dependent and aggregative movement of herbivores (Lewis 1994) or time lags in the decline or recovery of plant quality following herbivory (Turchin 2003; Underwood 1999) can produce sustained oscillations. By 

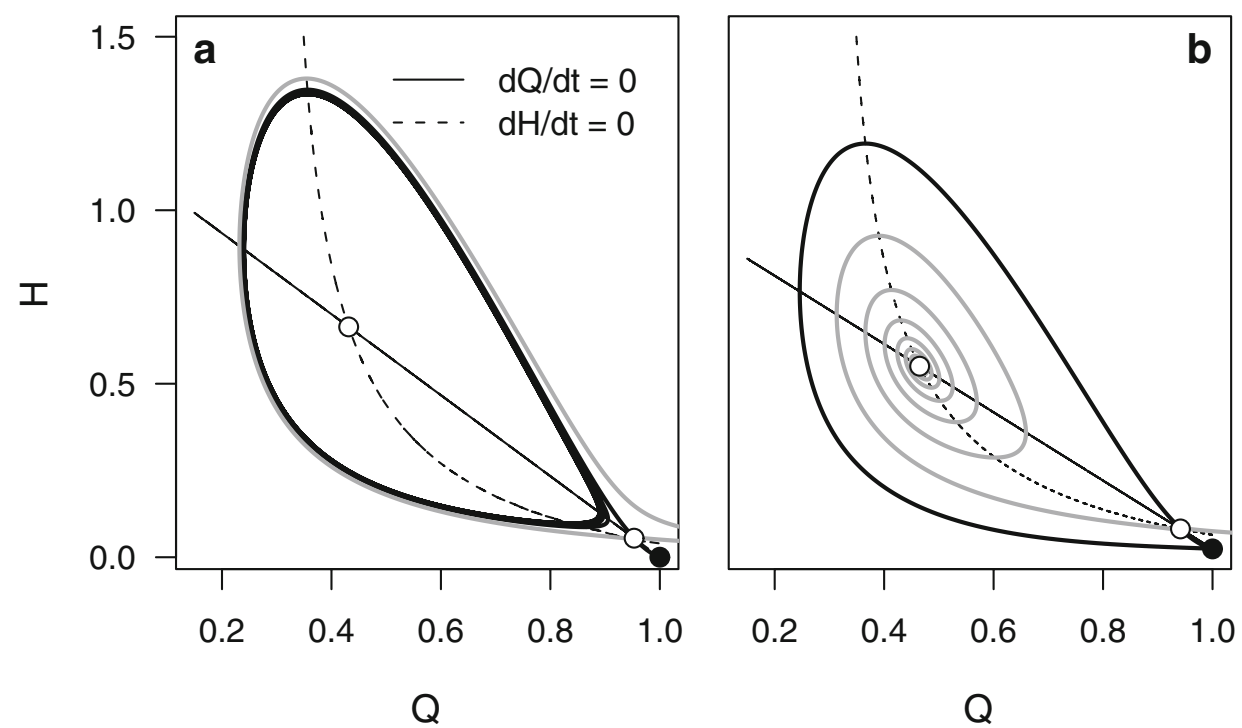

Fig. 7 Phase-plane diagrams illustrating the dynamics of a strongly obligate herbivore when the upper equilibrium is unstable. Composite parameter values are at their default values, with two exceptions: $m_{P}=$ 6.75 (see Fig. $5 \mathrm{~g}$ ) and in $\mathbf{A}, R_{Q}=0.58$. Note that decreasing $R_{Q}$ in $\mathbf{A}$ allows for the persistence of stable oscillations-inside the lower equilibrium's stable manifold (thick gray line), the lower equilibrium

is not encountered, and the unstable manifold (thick black line) is attracted to a stable limit cycle. B The unstable manifold of the upper equilibrium joins the stable manifold of the lower equilibrium to produce a heteroclinic orbit (thick gray line); all other starting points lead to herbivore extinction, including the unstable manifold of the lower equilibrium (thick black line)

explicitly including interactions with predators and mutualists, our model adds another factor (protection mutualism) that may increase the likelihood that plant quality feedback will drive persistent herbivore fluctuations. Specifically, the increased potential for local herbivore populations to grow under the protective umbrella provided by their mutualists may have the detrimental effect of allowing them to overexploit their host plants (e.g., by imposing nutritional stress or by inducing plant defenses), leading to herbivore decline followed by plant recovery and, eventually, a reinitiation of the cycle. Somewhat paradoxically, this sometimes leads to the situation in which the herbivore is both obligately dependent on its mutualist (as indicated by the existence of a lower, unstable equilibrium) and susceptible to extinction driven by high-amplitude oscillations made possible by the mutualist (Fig. 6b).

As in any modeling analysis, our conclusions may depend on the model assumptions. We assume that predation on herbivores is primarily via generalist predators and that mutualists act as generalists in their interactions with

herbivores. We formulated the increase of predators and mutualists to herbivores as a behavioral "aggregation response" with no numerical feedback on mutualist or predator population densities, arguing that this formulation applies for many protection mutualisms (cf. Buckley 1987; Hölldobler and Wilson 1990; Stadler and Dixon 2005). This approach is typical of the distinction between generalist and specialist predators in predator-prey-based consumer-resource models (Gilg et al. 2003; Turchin and Hanski 1997). That being said, few studies have explicitly tested the degree of benefit received by mutualists in protection mutualisms (Beattie 1991; Cushman and Beattie 1991), although a few studies of ant-protection mutualism suggest the potential for feedback dynamics, especially on densities of the worker population (Cushman et al. 1994; Cushman and Beattie 1991). Further analyses exploring the effect of adding numerical dynamics of the protector or mutualist, or of making consumption of resource by the herbivore a type II function, are warranted.

To simplify our analysis, we have not included a cost to herbivores from interacting with their protectors (Stadler and

Table 2 Percent of randomly chosen parameter combinations resulting in different dynamical outcomes for herbivores in models $2 \mathrm{a}-2 \mathrm{~d}$ and 4

\begin{tabular}{|c|c|c|c|c|c|}
\hline \multirow[t]{2}{*}{ Model } & \multirow[t]{2}{*}{ All equilibria $\leq 0$} & \multicolumn{2}{|c|}{$\begin{array}{l}1 \text { Positive equilibrium (facultative or weak obligate } \\
\text { mutualism) }\end{array}$} & \multicolumn{2}{|c|}{$\begin{array}{l}2 \text { Positive equilibria (strong obligate } \\
\text { mutualism) }\end{array}$} \\
\hline & & Stable & Unstable & Unstable/stable & Unstable/unstable \\
\hline$M^{*} P^{*} H Q$ (Eq. 4) & 7.1 & 59.1 & 31.0 & 2.4 & 0.4 \\
\hline$M P H Q$ (Eqs. 2a-2d) & 6.5 & 61.4 & 28.5 & 3.3 & 0.3 \\
\hline
\end{tabular}

Parameter sets were randomly sampled $(N=100,000)$ from the joint uniform distribution defined by the range $0-10$ times the baseline value of each parameter (Table 1) and applied to both models. At each parameter combination, equilibria and eigenvalues were computed numerically 
Dixon 1999) or a positive indirect effect of protectors on plants mediated by the removal of nonmutualist herbivores (reviewed in Styrsky and Eubanks 2007). Our assumptions are consistent with a number of ant-protection mutualisms (Flatt and Weisser 2000; Morales 2000b; Morales and Beal 2006; Stadler and Dixon 1999; Styrsky and Eubanks 2007), and we emphasize that relaxing these assumptions is not likely to change the conclusions reached here. For example, our results suggest that even if protectors increase plant quantity or quality over the short term by removing nonmutualist herbivores, herbivore population growth in response to elevated plant resource (in addition to protection from predators) over the longer term may cause plants, and then herbivores, to decline. In a preliminary analysis of a modification of Eq. 4 with an indirect positive effect of mutualists on plant quantity/quality, we have found that long-term oscillations are still possible (results not shown).

We conclude that mutualism can magnify the top-down effect of one partner on lower trophic levels and that this effect can engender oscillatory dynamics. The effect of herbivory on the subsequent performance of herbivores has been established for herbivore populations in general (Brown and Weis 1995; Root 1996; Uriarte 2000; Awmack and Leather 2002), and our results suggest that this effect is likely to be especially pronounced for herbivores that also engage in mutualisms. While the focus of protection mutualisms has understandably been on higher (i.e., predator) trophic levels, focusing on the effect of lower trophic levels will be an important new direction for future studies.

Acknowledgements The authors thank A. de Roos for helpful comments on an earlier draft. This work was supported by sabbatical funding from Williams College to MAM and by NSF Grant DEB0087096 to WFM.

\section{Appendix}

Stability of the baseline herbivore-quality model

Here, we show that nontrivial equilibria of the herbivorequality model without mutualism (three in the text) are strictly stable. Rewriting Eq. 3 as $d H / / d \tau=f(H, Q)$ and $d Q / d \tau=$ $g(H, Q)$ where $f(H, Q)=R_{T} Q H-m_{P} H(1+k H)$ and $g(H, Q)=R_{Q} Q(1-Q) h H Q$, equilibria $(H, Q)=(\bar{H}, \bar{Q})$ are defined by $f(\bar{H}, \bar{Q})=g(\bar{H}, \bar{Q})=0$. From the condition $f(\bar{H}, \bar{Q})=0$, we know that $r_{H} \bar{Q}-m_{P}-m_{P} k \bar{H}=0$, so the upper left term in the Jacobian matrix is

$$
\begin{aligned}
& \left.\frac{d f}{d H}\right|_{H, Q=\bar{H}, \bar{Q}}=r_{H} \bar{Q}-m_{P}-2 m_{P} k \bar{H} \\
& \quad=r_{H} \bar{Q}-m_{P}-m_{P} k \bar{H}-m_{P} k \bar{H}=-m_{P} k \bar{H}
\end{aligned}
$$

We also know from the condition $g(\bar{H}, \bar{Q})=0$ that $r_{Q}(1-\bar{Q})-h \bar{H}=0$, so the lower right term in the Jacobian is

$$
\begin{aligned}
\left.\frac{d g}{d Q}\right|_{H, Q=\bar{H}, \bar{Q}} & =r_{Q}(1-2 \bar{Q})-h \bar{H} \\
& =r_{Q}(1-\bar{Q})-h \bar{H}-r_{Q} \bar{Q}=-r_{Q} \bar{Q}
\end{aligned}
$$

The full Jacobian is

$\mathbf{J}=\left[\begin{array}{cc}-m_{P} k \bar{H} & r_{H} \bar{H} \\ -h \bar{Q} & -r_{Q} \bar{Q}\end{array}\right]$

which has trace $\operatorname{Tr} \mathbf{J}=-m_{P} k \bar{H}-r_{Q} \bar{Q}$ and determinant $\operatorname{det} \mathbf{J}=m_{P} r_{Q} k \overline{H Q}+r_{H} h \overline{H Q}$. Because the trace is negative and the determinant positive if $\bar{H}, \bar{Q}>0$, any nontrivial equilibrium is strictly stable.

Allee effect in herbivores resulting from protection mutualism

The defining feature of an Allee effect is that the herbivore's per-capita death rate as a function of $H$ has a negative slope at $H=0$. Differentiating the herbivore's percapita death rate in Eq. 4 and substituting $H=0$, one can see that the sign of the slope is the same as the sign of

$k\left(1+\frac{g}{d_{M}}\right)-a \frac{g}{d_{M}}$

If $a$ is sufficiently large, there will be an Allee effect increasing in strength with increasing $a$. Because $a$ governs the aggregation of protectors to herbivores, it is thus protector behavior that is directly generating the Allee effect in the herbivores.

We next explore the destabilizing influence of an Allee effect using a simpler version of Eq. 4, in which we replace the herbivore's per-capita death rate with its second-order Taylor series expansion, which captures its basic features. Specifically, let the herbivore's per-capita death rate $d(H)$ be a quadratic function $d(H)=\alpha-\beta H+\gamma H^{2}$, where $\alpha$ is the density-independent death rate, $\beta$ is the magnitude of the slope of $d(H)$ at $H=0$, and $\gamma$ governs the densitydependent increase in the death rate that dominates at high herbivore density because predators continuously arrive but never leave. By definition, there will be an Allee effect if $\beta>0$. Using $d(H)$, the analog of Eq. 4 is

$\frac{d H}{d \tau}=R_{H} Q H-\left(\alpha-\beta H+\gamma H^{2}\right) H$
$\frac{d Q}{d \tau}=R_{Q} Q(1-Q)-h H Q$ 
The Jacobian of Eq. 5, expressed in terms of the equilibrium values $\bar{H}$ and $\bar{Q}$, is

$J=\left[\begin{array}{lc}\beta \bar{H}-2 \gamma \bar{H}^{2} & R_{T} \bar{H} \\ -h \bar{Q} & -R_{Q} \bar{Q}\end{array}\right]$

A feasible equilibrium $(\bar{H}, \bar{Q})>0$ will be stable if $\operatorname{Tr}(J)$ $<0$ and $\operatorname{det}(J)>0$. If $\beta=\gamma=0$, Eq. 5 is equivalent to a model with a self-limiting prey $(Q)$ with type I consumption by a predator $(H)$ with density-independent death rate $\alpha$, and, as the stability conditions demonstrate, that model is strictly stable. Letting $\gamma>0$ with $\beta=0$ only adds more stability. However, if the Allee effect is sufficiently strong (specifically, if $\beta>2 \gamma \bar{H}-R_{Q} \bar{Q} / \bar{H}$, so that $\left.\operatorname{Tr}(J)>0\right)$, then $(\bar{H}, \bar{Q})$ will be an unstable equilibrium. Thus, in this simplified model it is only large, positive values for the parameter $\beta$ that generate instability, which, in turn, demonstrates that aggregation of protectors, which produces the Allee effect, is the ultimate source of instability.

Conditions for two equilibria

Here we show that the existence of two positive equilibria in Eq. 4 implies that the mutualism is strongly obligate from the perspective of the herbivore. From the nondimensionalized model (Eqs. 2a-2d), on a plant with $H=0$, the equilibrium mutualist density will be (setting Eq. 2a to zero) $M^{*}=1 / d_{M}$, and the equilibrium predator density will be (setting Eq. $2 b$ to zero)

$P^{*}=m_{P} \frac{1+k(0)}{1+g M^{*}}=\frac{m_{P}}{1+g / d_{M}}$

A herbivore population will not be able to establish on an uninduced plant (with $Q=1$ ) if the per-capita growth rate is negative, i.e., if (from Eq. 2c)

$R_{H}(1)-P^{*}=R_{H}-\frac{m_{P}}{1+g / d_{M}}<0$

Therefore, the condition for the mutualism to be strongly obligate (i.e., to persist, herbivores need more mutualists than would be present on a plant before any herbivores are present) is:

$m_{P}>R_{H}\left(1+g / d_{M}\right)$

Now, the model with rapidly equilibrating mutualists and predators (Eq. 4) has the following isoclines:

$$
\begin{aligned}
\frac{d H}{d \tau} & =0 \Rightarrow Q \\
& =\frac{m_{P}(1+H)(1+k H)}{R_{H}\left(1+g / d_{M}+\left(1+(1+a) g / d_{M}\right) H\right)}
\end{aligned}
$$

$\frac{d Q}{d \tau}=0 \Rightarrow Q=1-\left(h / R_{Q}\right) H$

From Eq. 7b, the $Q$ isocline always intercepts the $Q$ axis (where $H=0$ ) at $Q=1$. From Eq. $7 \mathrm{~b}$, the $H$ isocline intercepts the $Q$ axis at $Q=m_{P} / R_{H}\left(1+g / d_{M}\right)$. Therefore, if $m_{P} / R_{H}\left(1+g / d_{M}\right)>1$ or

$m_{P} / R_{H}\left(1+g / d_{M}\right)$

the $Q$ intercept of the $H$ isocline is above the $Q$ intercept of the $Q$ isocline, and the two isoclines will cross twice (e.g., Figs. 2A and 3). However, as Eq. 6 and 8 are the same, the condition for strong obligacy is the same as the condition for the existence of two equilibria. Finally, note that conditions 6 and 8 also indicate that strong obligacy and the existence of two equilibria, respectively, will be more likely if $R_{H}$ is small (Fig. 2a).

\section{References}

Addicott JF (1981) Stability properties of 2-species models of mutualisms. Oecologia 49:42-49

Armstrong R (1987) A patch model of mutualism. J Theor Biol 125:243-246

Awmack C, Leather S (2002) Host-plant quality and fecundity in herbivorous insects. Annu Rev Entomol 47:817-844

Axén A, Pierce N (1998) Aggregation as a cost-reducing strategy for lycaenid larvae. Behav Ecol 9:109-115

Beattie AJ (1991) Problems outstanding in ant-plant interaction research. In: Huxley C, Cutler D (eds) Ant-plant interactions. Oxford University Press, London, pp 559-576

Breton LM, Addicott JF (1992) Does host-plant quality mediate aphid-ant mutualism? Oikos 63:253-259

Bronstein JL, Barbosa P (2002) Multi-trophic/multi-species mutualistic interactions: the role of non-mutualists in shaping and mediating mutualisms. In: Hawkins B, Tscharntke T (eds) Multitrophic level interactions. Cambridge University Press, Cambridge, pp 44-65

Brown DG, Weis AE (1995) Direct and indirect effects of prior grazing of goldenrod upon the performance of a leaf beetle. Ecology 76:426-436

Buckley RC (1987) Interactions involving plants, Homoptera, and ants. Ann Rev Ecolog Syst 18:111-135

Cushman JH, Whitham TG (1989) Conditional mutualism in a membracid-ant association: temporal, age-specific, and densitydependent effects. Ecology 70:1040-1047

Cushman JH, Addicott JF (1989) Intra specific and interspecific competition for mutualists - ants as a limited and limiting resource for aphids. Oecologia 79:315-321

Cushman JH, Beattie AJ (1991) Mutualisms: assessing the benefits to hosts and visitors. Trends Ecol Evol 6:193-195

Cushman JH, Whitham TG (1991) Competition mediating the outcome of a mutualism: protective services of ants as a limiting resource for membracids. Am Nat 138:851-865

Cushman JH, Rashbrook VK, Beattie AJ (1994) Assessing benefits to both participants in a lycaenid-ant association. Ecology 75:1031-1041

Dean A (1983) A simple model of mutualism. Am Nat 121:409-417

DeVries P (1991) Evolutionary and ecological patterns in myrmecophilous riodinoid butterflies. In: Huxley C, Cutler D (eds) Antplant interactions. Oxford University Press, Oxford, pp 143-156 
Diehl S, Feißel M (2000) Effect of enrichment on three-level food chains with omnivory. Am Nat 155:200-218

Dyck H, Oostermeijer JGB, Talloen W, Feenstra V, van der Hidde, Wynhoff A (2000) Does the presence of ant nests matter for oviposition to a specialized myrmecophilous Maculinea butterfly? Proc R Soc Lond B Biol Sci 267:861-866

Edelstein-Keshet L, Rausher MD (1989) The effects of inducible plant defenses on herbivore populations. 1. Mobile herbivores In continuous-time. Am Nat 133:787-810

Ermentrout B (2002) Simulating, analyzing, and animating dynamical systems: a guide to XPPAUT for researchers and students. Society for Industrial and Applied Mathematics, Philadelphia

Finke DL, Denno RF (2004) Predator diversity dampens trophic cascades. Nature 429:407-410

Flatt T, Weisser WW (2000) The effects of mutualistic ants on aphid life history traits. Ecology 81:3522-3529

Fretwell S (1977) The regulation of communities by the food chains exploiting them. Perspect Biol Med 20:169-185

Gause G, Witt A (1935) Behavior of mixed populations and the problem of natural selection. Am Nat 69:596-609

Gilg O, Hanski I, Sittler B (2003) Cyclic dynamics in a simple vertebrate predator-prey community. Science 302:866-868

Hairston N, Smith F, Slobodkin L (1960) Community structure, population control, and competition. Am Nat 94:421-425

Halaj J, Wise D (2001) Terrestrial trophic cascades: how much do they trickle? Am Nat 157:262-281

Hastings A, Powell T (1991) Chaos in a three-species food chain. Ecology 72:896-903

Heithaus ER, Culver DC, Beattie AJ (1980) Models of some ant-plant mutualisms. Am Nat 116:347-361

Holland JN, DeAngelis DL (2001) Population dynamics and the ecological stability of obligate pollination mutualisms. Oecologia 126:575-586

Hölldobler B, Wilson EO (1990) The ants. Belknap, Cambridge

Hunter M, Price P (1992) Playing chutes and ladders: heterogeneity and the relative roles of bottom-up and top-down forces in natural communities. Ecology 73:724-732

Knight TM, Chase JM, Hillebrand H, Holt RD (2006) Predation on mutualists can reduce the strength of trophic cascades. Ecol Lett 9:1173-1178

Kooi BW, Kuijper LDJ, Kooijman SALM (2004) Consequences of symbiosis for food web dynamics. J Math Biol 49:227-271

Kuznetsov YA (1995) Elements of applied bifurcation theory. Springer, Berlin Heidelberg New York

Lewis MA (1994) Spatial coupling of plant and herbivore dynamicsthe contribution of herbivore dispersal to transient and persistent waves of damage. Theor Popul Biol 45:277-312

May R (1974) Stability and complexity in model ecosystems. Princeton University Press, Princeton

May RM (1976) Models for two interacting populations. In: May RM (ed) Theoretical ecology. Saunders, Philadelphia, pp 4970

May RM (1978) Mathematical aspects of the dynamics of animal populations. In: Levin S (ed) Studies in mathematical biology, vol 16. Part II. Populations and communities. Mathematical Association of America, New York, pp 317-366

McCann KS, Hastings A, Huxel G (1998) Weak trophic interactions and the balance of nature. Nature 395:794-798

Morales MA (2000a) Survivorship of an ant-tended membracid as a function of ant recruitment. Oikos 90:469-476

Morales MA (2000b) Mechanisms and density dependence of benefit in an ant-membracid mutualism. Ecology 81:482-489
Morales MA (2002) Ant-dependent oviposition in the membracid, Publilia concava. Ecol Entomol 27:247-250

Morales MA, Beal ALH (2006) The effects of host-plant quality and ant tending for the treehopper, Publilia concava. Ann Entomol Soc Am 99:545-552

Morris W, Dwyer G (1997) Population consequences of constitutive and inducible plant resistance: herbivore spatial spread. Am Nat 149:1071-1090

Morris WF, Bronstein JL, Wilson WG (2003) Three-way coexistence in obligate mutualist-exploiter interactions: the potential role of competition. Am Nat 161:860-875

Nisbet R, Diehl S, Wilson W, Cooper S, Donalson D, Kratz K (1997) Primary productivity gradients and short-term population dynamics in open systems. Ecol Monogr 67:535-553

Pierce N, Easteal S (1986) The selective advantage of attendant ants for the lycaenid butterfly, Glaucopsyche lygdamus. J Anim Ecol $55: 451-462$

Power M (1992) Top-down and bottom-up forces in food webs: do plants have primacy? Ecology 73:733-746

Ringel M, Hu H, Anderson G (1996) The stability and persistence of mutualisms embedded in community interactions. Theor Popul Biol 50:281-297

Risch S, Carroll C (1982) Effects of a keystone predaceous ant, Solenopsis geminata, on arthropods in a tropical agro-ecosystem. Ecology 63:1979-1983

Root RB (1996) Herbivore pressure on goldenrods (Solidago altissima): its variation and cumulative effects. Ecology 77:1074-1087

Schmitz O, Vlastimil K, Ovadia O (2004) Trophic cascades: the primacy of indirect interactions. Ecol Lett 7:153-163

Stadler B, Dixon AFG (1999) Ant attendance in aphids: why different degrees of myrmecophily? Ecol Entomol 24:363-369

Stadler B, Dixon AFG (2005) Ecology and evolution of aphid-ant interactions. Ann Rev Ecol Evol Syst 36:345-372

Stanton ML (2003) Interacting guilds: moving beyond a pairwise perspective. Am Nat 162:s10-s23

Styrsky JD, Eubanks MD (2007) Ecological consequences of interactions between ants and honeydew-producing insects. Proc Biol Sci 274:151-164

Turchin P (2003) Complex population dynamics: a theoretical/ empirical synthesis. Princeton University Press, Princeton

Turchin P, Hanski I (1997) An empirically based model for latitudinal gradient in vole population dynamics. Am Nat 149:842-874

Underwood N (1999) The influence of plant and herbivore characteristics on the interaction between induced resistance and herbivore population dynamics. Am Nat 153:282-294

Uriarte M (2000) Interactions between goldenrod (Solidago altissima L.) and its insect herbivore (Trirhabda virgata) over the course of succession. Oecologia 122:521-528

Vandermeer J, Boucher D (1978) Varieties of mutualistic interactions in population models. J Theor Biol 74:549-558

Way M (1963) Mutualism between ants and honeydew-producing Homoptera. Annu Rev Entomol 8:307-344

Wimp G, Whitham TG (2001) Biodiversity consequences of predation and host-plant hybridization on an aphid-ant mutualism. Ecology 82:440-452

Wolin CL (1985) The population dynamics of mutualistic systems. In: Boucher DH (ed) The biology of mutualism. Oxford University Press, New York, pp 40-99

Wolin C, Lawlor L (1984) Models of facultative mutualism: density effects. Am Nat 124:843-862

Wright DH (1989) A simple stable model of mutualism incorporating handling time. Am Nat 134:664-667 